\title{
CARACTERÍSTICAS CLIINICAS Y VALORACIÓN GERIÁTRICA EN ADULTOS MAYORES CON NEUMONÍA DE UN HOSPITAL DE BUENOS AIRES, ARGENTINA
}

\author{
Martín Regueiro ${ }^{1,2,3, a}$, Cecilia Homar ${ }^{1,2, a}$, Ricardo Ovejero ${ }^{2, a}$, Antonio Liü,a, \\ Tomás Pieroni2,a, Romina Basilico 2 ,a
}

\begin{abstract}
RESUMEN
Con el objetivo de determinar las características clínicas y geriátricas en pacientes adultos mayores con neumonía, en un hospital de Buenos Aires en Argentina, se realizó un estudio transversal en el 2010, con 66 pacientes hospitalizados por neumonía. La edad media fue de 82 años, siendo el 54,5\% hombres. El 47,0\% de los ingresos fueron por neumonía adquirida en la comunidad. En cuanto a la valoración funcional el 31,8\% eran dependientes para las actividades básicas de la vida diaria y $69,4 \%$ dependientes para actividades instrumentales de la vida diaria. Las características clínicas más frecuentes fueron fiebre, tos, disnea y expectoración. Se identificó el agente causal de la neumonía en el 34,8\% de pacientes, siendo 18 bacterianas $(27,2 \%$ ) y cinco virales $(7,5 \%)$. La mortalidad cruda fue del $28,8 \%$, pero cercana al $50 \%$ en los que tenían síndrome confusional. Se concluye que la neumonía en adultos mayores tiene características especiales que se hacen visibles con una correcta valoración diagnóstica y geriátrica.
\end{abstract}

Palabras clave: Neumonía; Anciano; Etiología; Geriatría (fuente: DeCS BIREME).

\section{CLINICAL CHARACTERISTICS AND GERIATRIC EVALUATION OF ELDERLY PATIENTS WITH PNEUMONIA IN A HOSPITAL IN BUENOS AIRES, ARGENTINA}

\begin{abstract}
In order to determine the clinical and geriatric characteristics of elderly adults with pneumonia in a hospital in Buenos Aires, Argentina, a cross-sectional study was carried out in 2010, included 66 patients hospitalized for pneumonia. The mean age of the study subjects was $82,54.5 \%$ of whom were men. $47.0 \%$ of the hospitalizations were due to community-acquired pneumonia. As for the functional evaluation, 31.8\% showed dependence in performing basic activities of daily living and $69.4 \%$ presented dependence in instrumental activities of daily living. The most frequent clinical characteristics observed were fever, cough, dyspnea and expectoration. The causative agent of pneumonia was identified in $34.8 \%$ of the cases, 18 of which were bacterial $(27.2 \%)$ and 5 viral $(7.5 \%)$. The crude mortality rate was $28.8 \%$, but was close to $50 \%$ in those patients having confusional syndrome. We conclude that pneumonia in elderly patients has special characteristics, which can be observed with a proper diagnostic and geriatric evaluation.
\end{abstract}

Key words: Pneumonia; Aged; Etiology; Geriatrics (source: MeSH NLM).

\section{INTRODUCCIÓN}

La neumonía es una infección aguda del parénquima pulmonar que se manifiesta por signos y síntomas de infección respiratoria baja, asociados a un nuevo infiltrado en la radiografía de tórax ${ }^{(1)}$. Esta infección compromete a todos los grupos de edad, aunque los adultos mayores tienen mayor vulnerabilidad en sus formas graves vinculada con la morbilidad; el medio sociosanitario, y el estado inmune y nutricional previo ${ }^{(2)}$. La sintomatología en adultos mayores puede variar presentándose como un estado confusional, con pérdida de su capacidad funcional o con descompensación de su enfermedad de base.

A pesar del impacto en salud pública, y de ser mayor su incidencia en adultos mayores, existen escasos datos sobre la etiología de la neumonía en este grupo de personas y, en particular la de causa viral. A la hora de evaluar un paciente adulto mayor con neumonía,

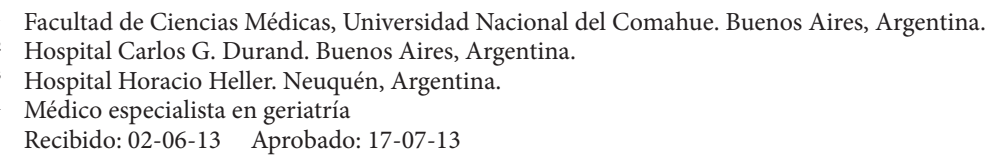

Citar como: Regueiro M, Homar C, Ovejero R, Liu A, Pieroni T, Basilico R. Características clínicas y valoración geriátrica en adultos mayores con neumonía de un hospital de Buenos Aires, Argentina. Rev Peru Med Exp Salud Publica. 2013;30(3):432-6. 
la valoración geriátrica integral y las herramientas de valoración de gravedad tienen un rol fundamental, con mayor aproximación diagnóstica cuando son usadas juntas. Se considera adecuado que en la atención del adulto mayor internado se considere su estado funcional, cognitivo y nutricional para la toma de decisiones ${ }^{(3)}$.

El presente estudio tiene como objetivo determinar las características clínicas y geriátricas de los pacientes con neumonía de un hospital de Buenos Aires, Argentina.

\section{EL ESTUDIO}

Estudio observacional y transversal en el que participaron pacientes mayores de 65 años de edad con diagnóstico de neumonía durante el año 2010 y que requirieron internación o estaban internados en la Unidad de Geriatría del Hospital Carlos G. Durand de Buenos Aires, Argentina.

El diagnóstico de neumonía fue definido según criterios del estudio PORT (3) considerando la aparición de algún criterio mayor entre fiebre, tos y expectoración o bien, dos criterios menores entre disnea, dolor pleurítico, síndrome confusional, signos de consolidación y leucocitosis mayor de 12 000/. $\mathrm{mm}^{3}$, asociado a la aparición de un infiltrado nuevo en la radiografía de tórax o la progresión de uno previo que no puede explicarse por otra causa. Según su procedencia, los tipos de neumonía se clasificaron en neumonía adquirida en la comunidad (NAC); neumonía intrahospitalaria (NIH), y neumonía de medio sanitario (NMS). Se consideró NIH a la que comienza después de 48 horas de ingreso hospitalario o dentro de las dos semanas posteriores al alta; NMS es aquella que aparece en individuos procedentes de hospitales de día, residencias o que hayan estado internados durante al menos 48 horas en los últimos 90 días previos a los síntomas, recibieron quimioterapia, diálisis o curas en domicilio en los últimos 30 días ${ }^{(2,4)}$.

Se recopilaron los siguientes datos al ingreso: edad, sexo, residencia en centro geriátrico o domicilio familiar. Se consideraron las siguientes comorbilidades: hipertensión arterial, deterioro cognitivo, enfermedad pulmonar obstructiva crónica, diabetes mellitus, enfermedad cerebrovascular, Párkinson, neoplasias, hepatopatía, cardiopatía isquémica, insuficiencia cardíaca, insuficiencia renal y $\mathrm{VIH}$.

La valoración geriátrica integral se realizó usando índice de Katz ${ }^{(5)}$ para actividades básicas de la vida diaria (ABVD) y el índice de Lawton-Brody ${ }^{(6)}$ para actividades instrumentales de la vida diaria (AIVD) para definir el estado funcional. Se consideró dependencia para ABVD con Katz mayor de 1 y dependencia para AIVD con Lawton-Brody menor de 8 en mujeres o 5 en hombres. La valoración cognitiva por minimental se realizó a las 48 horas de la internación en los pacientes que tenían CAM (Confusion Assessment Method) negativos, ajustado a edad y nivel educativo según puntos de corte nacionales ${ }^{(7,8)}$. El CAM es un método sencillo que permite diagnosticar síndrome confusional ${ }^{(9)}$.

Con respecto a los datos clínicos, se registró la presencia de tos, expectoración, cefalea, dolor abdominal, fiebre, escalofríos, dolor torácico, disnea, y confusión. Se midió presión arterial, pulso, frecuencia respiratoria, disnea, cianosis, estertores crepitantes y signos de consolidación o exploración normal.

En el laboratorio se realizó dos hemocultivos e hisopado nasofaríngeo para diagnóstico de virus, entre los que se cuentan los virus influenza $A$, influenza $B$, adenovirus, parainfluenza 1, 2 o 3; y metapneumovirus. El estudio consideró como puntos de terminación de seguimiento el alta médica, la pérdida por derivación o alta voluntaria, y el fallecimiento.

Como predictores de mortalidad se usaron las escalas de CRB65 (11,12); CURB65 y PSI (13). Estas reglas de predicción permiten estimar mortalidad y necesidad de internación. PSI (Pneumonia Severity Index) considera 20 variables mientras que el CURB65 y CRB65 considera solo 4 o 5 variables haciéndolas más amigables y rápidas de realizar. Incluye confusión, frecuencia respiratoria $>30$ por minuto, presión arterial $<$ 90-60 mmHg, y edad > 65. Mientras que CURB65 incluye úrea mayor $42 \mathrm{mg} / \mathrm{dL}$.

Solo se incluyeron las personas que accedieron a participar del estudio luego de firmar el consentimiento informado, el cual podía ser firmado por el paciente o sus familiares a cargo.

\section{HALLAZGOS}

Se incluyó a 66 pacientes adultos mayores, la edad media en años de los pacientes fue de $82 \pm 8$ (65-99). Del total de pacientes 36 (54,5\%) fueron hombres. La procedencia de los tipos de neumonía fue en su mayoría NAC (47\%), seguido de las NIH (28,8\%) y las NMS (24,2\%). Del total de neumonías $28,8 \%$ fueron aspirativas. Veintiún pacientes $(31,8 \%)$ eran dependientes para las ABVD; mientras que 25 (69,4\%) eran dependientes para AIVD. Las características de la población y sus comorbilidades se detallan en 
Tabla 1. Características epidemiológicas al ingreso en adultos mayores hospitalizados por neumonía, Buenos Aires, 2010

\begin{tabular}{lcc}
\hline \multicolumn{1}{c}{ Características } & N. ${ }^{\circ}$ & $(\%)$ \\
\hline Edad $^{*}$ & $82 \pm 8$ \\
Sexo & & \\
$\quad$ Hombre & 36 & $(54,5)$ \\
\hline Mujer & 30 & $(45,5)$ \\
\hline Residencia & & \\
\hline Domicilio familiar & 47 & $(71,2)$ \\
\hline Centro geriátrico & 19 & $(28,8)$ \\
\hline Tipo de neumonía & & \\
\hline Neumonía adquirida en la comunidad & 31 & $(47,0)$ \\
\hline Neumonía intrahospitalaria & 19 & $(28,8)$ \\
\hline Neumonía de medio sanitario & 16 & $(24,2)$ \\
\hline Valoración geriátrica integral & & \\
\hline Dependientes para ABVD & 21 & $(31,8)$ \\
\hline Dependientes para AIVD & 25 & $(69,4)$ \\
\hline Comorbilidades ${ }^{\dagger}$ & & \\
\hline Hipertensión arterial & 43 & $(65,2)$ \\
\hline Deterioro cognitivo & 30 & $(45,5)$ \\
\hline Insuficiencia cardíaca & 24 & $(36,9)$ \\
\hline EPOC & 20 & $(30,3)$ \\
\hline Enfermedad cerebro vascular & 19 & $(28,8)$ \\
\hline Cardiopatía isquémica & 14 & $(21,2)$ \\
\hline Neoplasias & 12 & $(22,7)$ \\
\hline Diabetes & 9 & $(13,6)$ \\
\hline Insuficiencia renal & 8 & $(12,2)$ \\
\hline Parkinson & 3 & $(4,5)$ \\
\hline Hepatopatía & 1 & $(1,5)$ \\
\hline
\end{tabular}

* Media \pm desviación estándar.

† La suma de categorías no es igual al " $n$ " de la muestra porque un paciente puede tener más de una comorbilidad.

ABVD: actividades básicas de la vida diaria, AIVD: actividades instrumentales de la vida diaria, EPOC: enfermedad pulmonar obstructiva crónica

Tabla 1. Las características clínicas más frecuentes fueron fiebre, tos, disnea y expectoración. Según los resultados de laboratorio se presentó leucocitosis mayor de 12 000/mm3 y úrea mayor de 45 mg/dL en el $65,1 \%$ de los pacientes (Tabla 2 ).

La valoración cognitiva mínima que se realizó en los pacientes evidenció que $28(43,1 \%)$ de los 66 pacientes estudiados tenían CAM positivo, compatible con diagnóstico de síndrome confusional. De los 38 restantes, $14(37,8 \%)$ tenían minimental menor de 21 , compatible con deterioro cognitivo. En la Tabla 3 se detalla el resto de características geriátricas de la población estudiada.

Los esquemas de vacunación estaban incompletos en el $100 \%$ de la población según las normas nacionales de vacunación actualizadas a la fecha de realización del estudio ${ }^{(14)}$; estando solo vacunados para influenza 8 $(12,3 \%)$ y para neumococo $5(7,7 \%)$ pacientes.
Tabla 2. Características clínicas y de laboratorio de los adultos mayores hospitalizados por neumonía, Buenos Aires, 2010

\begin{tabular}{|c|c|c|}
\hline Características & $\mathbf{N} .^{\circ}$ & (\%) \\
\hline \multicolumn{3}{|l|}{ Clínicas } \\
\hline Fiebre & 47 & $(72,3)$ \\
\hline Tos & 47 & $(72,3)$ \\
\hline Disnea & 45 & $(69,2)$ \\
\hline Expectoración & 40 & $(61,5)$ \\
\hline Hipoxemia & 18 & $(27,2)$ \\
\hline PAS $<90$ & 7 & $(10,8)$ \\
\hline Dolor pleurítico & 4 & $(6,2)$ \\
\hline \multicolumn{3}{|l|}{ Radiológicas } \\
\hline Rx de tórax con infiltrado $\geq 2$ lóbulos & 29 & $(43,9)$ \\
\hline $\mathrm{Rx}$ tórax con infiltrado bilateral & 10 & $(15,2)$ \\
\hline \multicolumn{3}{|l|}{ Laboratorio } \\
\hline Leucocitosis $\left(>12000 / \mathrm{mm}^{3}\right)$ & 43 & $(65,1)$ \\
\hline Urea (> 45mg/dL) & 43 & $(65,1)$ \\
\hline Hiponatremia (<135mEq/L) & 19 & $(28,7)$ \\
\hline Leucopenia $\left(<4000 / \mathrm{mm}^{3}\right)$ & 2 & $(3,0)$ \\
\hline
\end{tabular}

Se identificó el agente causal en 23 pacientes $(34,8 \%) ; 18$ fueron bacterianas $(27,2 \%)$ y 5 virales $(7,5 \%)$. Los agentes etiológicos más frecuentes fueron Streptococcus pneumoniae, Klebsiella pneumoniae y bacilos Gram negativos. Los virus respiratorios aislados fueron virus sincicial respiratorio, virus influenza $A$ y virus parainfluenza 3 . De los pacientes con neumonías bacterianas con germen identificado fallecieron seis pacientes $(33,3 \%)$ mientras que en el caso de neumonías virales no hubo fallecidos. El score de Fine (PSI) de los pacientes con neumonía bacteriana fue en promedio de 134,3 puntos mientras que si la etiología era viral el puntaje promedio fue de 122,5.

Los pacientes residentes en un domicilio familiar tenían 82 años en promedio, de los cuales fallecieron 11 pacientes $(23,4 \%)$. En este grupo se aisló el germen en 19 pacientes (42,2\%); siendo Streptococcus pneumoniae y virus respiratorios los más frecuentes con cuatro pacientes $(8,9 \%)$ cada uno, seguido por

Tabla 3. Características geriátricas de los adultos mayores hospitalizados por neumonía, Buenos Aires, 2010

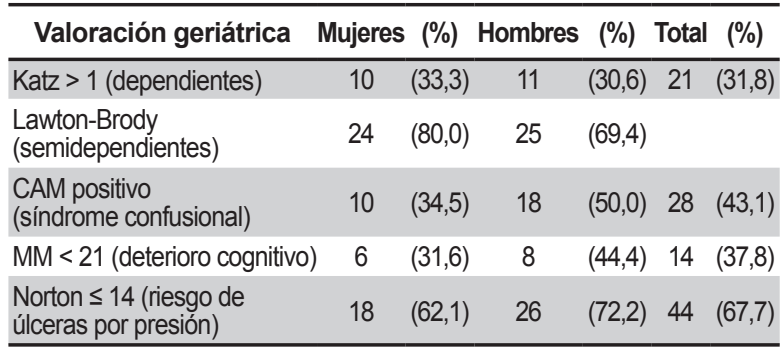

CAM: Confusion Assessment Method, MM: Minimental 
bacilos Gram negativos con tres pacientes (6,7\%). Los pacientes residentes en un centro geriátrico tenían una edad promedio de 83,5 años, de los cuales fallecieron cuatro $(21,1 \%)$. El agente etiológico se aisló en cuatro pacientes $(21 \%)$, siendo los gérmenes Streptococcus pneumoniae, Enterococo spp., Proteus mirabilis y virus sincitial respiratorio.

En cuanto a los predictores de mortalidad en promedio los pacientes tenían CRB65 de 1,7, CURB65 de 2,4 y PSI de 137,9. En nuestro estudio fallecieron 19 pacientes $(28,8 \%)$ en relación a los predictores antes mencionados. La mortalidad fue $9 / 31$ (29\%) en NAC; $6 / 19(31,6 \%)$ en $\mathrm{NIH} ; 4 / 16(25 \%)$ en NMS, y $7 / 19$ $(36,8 \%)$ cuando era aspirativa. Los pacientes que en el ingreso presentaban síndrome confusional tuvieron una mortalidad de $44,8 \%$ contra un $15,8 \%$ de los que tenían CAM negativo.

\section{DISCUSIÓN}

El estudio evidenció que la población adulta mayor hospitalizada en este hospital tenía un alto grado de dependencia en sus actividades básicas e instrumentales de la vida diaria. El porcentaje de pacientes con cuadro confusional diagnosticado por CAM fue semejante a otros estudios ${ }^{(16,17) .}$

Diversos estudios indican que la presentación habitual en pacientes jóvenes con la tríada típica de fiebre, tos productiva y dolor pleurítico no está presente en este grupo de edad, principalmente en aquellos con deterioro funcional o cognitivo ${ }^{(13,15,16)}$. En nuestro estudio el dolor pleurítico se evidenció en $6,2 \%$ de los pacientes, y la tríada completa no se encontró en ninguno de los 66 pacientes estudiados. Sin embargo, la mayoría de los pacientes tuvieron fiebre y tos con un $72,3 \%$ cada uno; seguido por disnea y expectoración con un $69,2 \%$ y un $61,5 \%$, respectivamente. Estos datos son semejantes a los encontrados en el estudio de Johnson donde constató fiebre, tos, disnea y expectoración en un 53$71 \%$; $61-67 \%$; $46-71 \%$ y $52 \%$ respectivamente ${ }^{(15)}$.

En otros estudios, como el de Ruiz en España, se evidenció fiebre, tos, disnea y expectoración en 38; 78; 69 y $61 \%$ de los pacientes respectivamente. La población estudiada en ese trabajo tenía una media de edad de 68 años, no incluía pacientes con $\mathrm{NIH}$, pero sí incluyo pacientes residentes en geriátricos ${ }^{(16)}$. Estos datos hacen suponer que a pesar de que la tríada completa no es frecuente en el grupo de edad, una neumonía sin fiebre, sin tos, sin disnea y/o sin expectoración, posiblemente no sea una neumonía.
Al evaluar los agentes etiológicos surge como primer punto crítico la falta de inclusión de la serología para microorganismos como chlamydia o mycoplasma, sin embargo, el presente estudio incluyo técnicas usadas en nuestro hospital en la entidad nosológica y en el grupo de edad. No se realizó toma de muestra de esputos por la cuestionada utilidad de la prueba y el alto porcentaje de pacientes con deterioro cognitivo que no podían realizar una correcta técnica para recolectar la muestra ${ }^{(10)}$.

Al considerar los virus, se demuestra su baja frecuencia en pacientes de edad avanzada. Entre los pacientes con neumonía viral no hubo fallecidos, fueron de menor gravedad al ingreso y mejor pronóstico. La frecuencia fue de $7,5 \%$, más baja que la encontrada en otros estudios, aunque los mismos utilizaban como método diagnóstico el par serológico ${ }^{(17,18)}$.

La población estudiada tenía, al ingreso, una predicción de mortalidad por score de Fine y CURB65 elevada. Estas características de predicción y mortalidad coinciden con otros estudios que avalan el uso de estas herramientas en la evaluación inicial del paciente con neumonía, y en especial en los adultos mayores.

El estado de vacunación completa fue bajo, teniendo en cuenta que el presente estudio se realizó un año después de la pandemia de gripe ocurrida en el año 2009. El 12\% de los pacientes estaban vacunados para influenza, siendo bajo también para neumococo. Surgen distintas interpretaciones de las bajas coberturas de vacunación de la población adulta mayor estudiada. Por un lado, existe evidencia del modesto beneficio de la vacuna de la gripe en sanos para disminuir los síntomas y la pérdida de días laborales, sin impacto sobre la disminución de las complicaciones por neumonía ${ }^{(19)}$. En cambio existen evidencias que demuestran los beneficios de revacunar a los adultos mayores contra tétanos y difteria cada 10 años, como se evidencia en la reaparición de difteria en Rusia durante los años 90 (20).

Esta situación se asocia a que no se cumplen las medidas de prevención en adultos mayores por inercia o por falta de evidencia sobre la terapéutica en el grupo de edad. Surge evidentemente un interrogante que no puede ser respondido por este trabajo relacionado a las ventajas de la vacuna y las medidas de prevención en este grupo de edad.

El estudio presenta limitaciones propias del diseño de investigación y de la selección de los pacientes lo que hace que estos resultados solo permitan sacar conclusiones respecto a grupos poblaciones con características similares. 
Concluimos que la neumonía en adultos mayores tiene características especiales que se hacen visibles con una correcta valoración diagnostica y geriátrica, siendo necesaria una mayor cobertura de las medidas de prevención disponibles en nuestro medio.

\begin{abstract}
Agradecimientos: a los médicos Florencia Hara, Nelly Vaca Lozada, Juan Pablo Linzitto, Cecilia Volpe y a la residencia de geriatría del Hospital Carlos G. Durand. También agradecemos al excelente servicio de enfermería y a los médicos de planta de la unidad de geriatría, al personal de laboratorio, especialmente a la bioquímica Lorena Lopez de virología y al Servicio de Nutrición del Hospital Carlos G. Durand. La asistencia técnica de Martín Cañás y Lorena Harrington.
\end{abstract}

Contribuciones de autoría: MR participó en la concepción y diseño del estudio. $\mathrm{CH}, \mathrm{RO}, \mathrm{AL}, \mathrm{TP}, \mathrm{RB}$ participaron en la recolección, análisis e interpretación de los resultados. $\mathrm{CH}$, $\mathrm{RO}, \mathrm{AL}, \mathrm{RB}$ participaron en la revisión crítica del artículo. TP participó en la aprobación de la versión final y obtención de financiamiento.

Fuentes de financiamiento: A cargo de los autores y del Hospital Carlos G. Durand.

Conflictos de interés: los autores declaran no tener conflictos de interés.

\section{REFERENCIAS BIBLIOGRÁFICAS}

1. Luna CM, Calmaggi A, Caberloto O, Gentile J, Valentini R, Ciruzzi J, et al. Neumonía adquirida en la comunidad. Guía Práctica elaborada por un comité intersociedades. Medicina (B Aires). 2003;63(4):319-43.

2. Pascual Rodríguez C, Alfageme Michavila I. Guía de la buena práctica clínica en geriatría: Neumonías. Madrid: Sociedad Española de Geriatría y Gerontología; 2006.

3. Fine MJ, Stone RA, Singer DE, Coley CM, Marrie TJ, Lave JR, et al. Processes and outcomes of care for patients with community-acquired pneumonia: results from the Pneumonia Patient Outcomes Research Team (PORT) cohort study. Arch Intern Med. 1999;159(9):970-80.

4. Tablan OC, Anderson LJ, Besser R, Bridges C, Hajjeh R; CDC; et al. Guidelines for preventing healthcare-associated pneumonia, 2003: recommendations of the $\mathrm{CDC}$ and the Health-care Infection Control Practices Advisory Committee. MMWR Recomm Rep. 2004;53(RR3):1-36.

5. Katz S, Branch LG, Branson $\mathrm{MH}$, Papsidero JA, Beck JC, Greer DS, et al. Active life expectancy. N Engl J Med. 1983;309(20):1218-24.

6. Lawton MP, Brody EM. Assessment of older people: self-maintaining and instrumental activities of daily living. Gerontologist. 1969;9(3):179-86.

7. Butman J, Arizaga RL, Harris P, Drake MA, Baumann D, de Pascale A, et al. El "Mini Mental State Examination” en Español. Normas para Buenos Aires. Rev Neurol Arg. 2001;26(1):11-5.
8. El "Mini-Mental State Examination" en la Argentina: Instrucciones para su administración. Grupo de Trabajo de Neuropsicología Clínica de la Sociedad Neurológica Argentina. Rev Neurol Arg. 1999;24(1):31-5.

9. Wei LA, Fearing MA, Sternberg EJ, Inouye SK. The Confusion Assessment Method: a systematic review of current usage. $\mathrm{J}$ Am Geriatr Soc. 2008;56(5):823-30. doi: 10.1111/j.1532-5415.2008.01674.x.

10. Ewig S, Schlochtermeier M, Göke N, Niederman MS. Applying sputum as a diagnostic tool in pneumonia: limited yield, minimal impact on treatment decisions. Chest. 2002;121(5):1486-92.

11. Lim WS, van der Eerden MM, Laing R, Boersma WG, Karalus N, Town GI, et al. Defining community acquired pneumonia severity on presentation to hospital: an international derivation and validation study. Thorax. 2003;58(5):377-82.

12. Bauer TT, Ewig S, Marre R, Suttorp $\mathrm{N}$, Welte T; CAPNETZ Study Group. CRB-65 predicts death from community-acquired pneumonia. J Intern Med. 2006;260(1):93-101.

13. Fine MJ, Smith MA, Carson CA, Mutha SS, Sankey SS, Weissfeld LA, et al. Prognosis and outcome of patients with community-acquired pneumonia. A metaanalysis. JAMA. 1996;275(2):134-41.

14. Argentina, Ministerio de Salud. Calendario Nacional de Vacunación de la República Argentina 2010 [Internet]. Buenos Aires: Ministerio de Salud [citado el 16 de marzo del 2011]. Disponible en: http://msal.gov.ar/ htm/Site/vacuna._cal2.asp
15. Johnson JC, Jayadevappa R, Baccash PD, Taylor L. Nonspecific presentation of pneumonia in hospitalized elderly people: age effect or dementia? J Am Geriatr Soc. 2000;48(10):1316-20.

16. Ruiz M, Ewig S, Marcos MA, Martinez JA, Arancibia F, Mensa J, et al. Etiology of community-acquired pneumonia: impact of age, comorbidity, and severity. Am J Respir Crit Care Med. 1999;160(2):397-405.

17. Starczewski AR, Allen SC, Vargas E, Lye M. Clinical prognostic indices of fatality in elderly patients admitted to hospital with acute pneumonia. Age Ageing. 1988;17(3):181-6.

18. de Roux A, Marcos MA, Garcia E, Mensa J, Ewig S, Lode H, et al. Viral community-acquired pneumonia in nonimmunocompromised adults. Chest. 2004;125(4):1343-51.

19. Jefferson T, Di Pietrantonj C, Rivetti A, Bawazeer GA, Al-Ansary LA, Ferroni E. Vaccines for preventing influenza in healthy adults. Cochrane Database Syst Rev. 2010;(7):CD001269. doi: 10.1002/14651858.CD001269.

20. Vitek CR, Wharton M. Diphtheria in the former Soviet Union: reemergence of a pandemic disease. Emerg Infect Dis. 1998;4(4):539-50.

Correspondencia: Martín Regueiro Dirección: Italia 476- CP 8324. Argentina Teléfono: 54-299-464200

Correo electrónico: regueiromartin@gmail.com 